\title{
SINR-based scheduling in multi-path multi-hop multi-radio multi-channel mmWave WPANs
}

\author{
Yunfeng Liu ${ }^{1}$, Zhiyong Feng ${ }^{1 *}$ (D) Zhiqing Wei ${ }^{1}$ and Zebing Feng ${ }^{2}$
}

\begin{abstract}
Millimeter wave (mmWave) communications is a prospective candidate technology for multi-gigabit rates multimedia applications. To combat the severe propagation attenuation of mmWave, the high gain directional antenna is commonly employed at the nodes. Moreover, exploiting multiple radios over multiple channels is also a promising technology to improve the throughput and delay performance of mmWave communications. In this paper, considering the signal to interference plus noise ratio (SINR) constraint, we develop a multi-path multi-hop multi-radio multi-channel (MPMH-MRMC) concurrent transmission scheduling algorithm to fully exploit spatial reuse in mmWave wireless personal area networks (WPANs). The problem of MPMH-MRMC scheduling is formulated as a mixed-integer linear programming (MILP) to clear all the flows within a minimum number of time slots, which is generally NP-hard. We further propose a heuristic MPMH-MRMC scheme with low computational complexity to solve the problem. Finally, through extensive simulations, we demonstrate that MPMH-MRMC can significantly improve the network performance in terms of network throughput and transmission delay under various traffic patterns.
\end{abstract}

Keywords: Multi-path multi-hop (MPMH) scheduling, Multi-radio multi-channel (MRMC) network, Spatial reuse, Wireless personal area networks (WPANs)

\section{Introduction}

Over the past decade, Millimeter wave (mmWave) communications in the $60 \mathrm{GHz}$ band has gained considerable attention from academia, industry, and standards bodies. Due to the unprecedented $7 \mathrm{GHz}$ unlicensed spectrum, mmWave communications is able to support multi-gigabit wireless multimedia services, such as uncompressed high-definition TV, instantaneous music, and image data transmission. With the recent advances in mmWave transceivers design [1], considerable interests have shown in standardizing specifications for mmWave systems, including IEEE 802.15.3c and IEEE 802.11 VHT [2].

Since the free space propagation loss scales as the square of the carrier frequency, the mmWave communications in the $60 \mathrm{GHz}$ band suffers severe attenuation. The free

\footnotetext{
${ }^{*}$ Correspondence: fengzy@bupt.edu.cn

${ }^{1}$ Key Lab. of Universal Wireless Communications Ministry of Education, Beijing University of Posts and Telecommunications (BUPT), Haidian Dist. Xitucheng Rd., Beijing, People's Republic of China
}

Full list of author information is available at the end of the article space propagation loss at $60 \mathrm{GHz}$ is $28 \mathrm{~dB}$ more than that at $2.4 \mathrm{GHz}$ [3]. Therefore, the mmWave communications range is limited, and it is mainly applied in shortrange indoor communications, such as wireless personal area networks (WPANs). To combat the severe propagation loss, the high gain directional antenna is commonly implemented at both the transmitter and receiver. As a result of directional listening and transmission, the deafness problem occurs when applying the carrier sense to avoid contentions within current transmissions. Besides, the multi-user interference (MUI) is relatively low, thus more concurrent transmissions can be supported to further increase the network capacity [4]. However, multiple communication links transmitting in the same time slot would lead to a high MUI, which might decrease the network throughput. Further, it is beneficial to employ multihop for transmission when the direct link is suffering low channel quality $[1,5]$. Therefore, how to schedule appropriate concurrent transmissions to improve the network throughput is a significant and challenging issue. 
The problem of concurrent transmission scheduling for mmWave communications has been investigated in the literatures. Most of the existing works focus on the singlepath single-hop single-radio single-channel (SPSH-SRSC) scenario [2-4, 6, 7]. A flip-based heuristic scheduling algorithm was proposed in [3] to maximize the number of flows scheduled in the network such that the quality of service (QoS) requirement of each flow was satisfied. In [4], Z. Yan et al. transformed the concurrent transmission maximization problem for one time slot into finding the feasible maximum clique of the conflict graph. In [8], L. Zhou et al. extended the scenario to multi-radio multi-channel (MRMC) network, which was mapped into a simple virtual SRSC network. A multi-path multihop (MPMH) scheduling scheme was proposed in [1], where the traffic across links of low channel quality was transmitted through MPMH to take advantage of spatial reuse.

Links in the mmWave network can be regarded as pseudo-wired, i.e., interference is negligible [9]. Motivated by this fact, we propose a novel MPMH-MRMC scheduling scheme to boost the spatial reuse in mmWave WPANs in this paper. By transmitting the traffic of flows with high traffic demand or low channel quality between the transmitters and receivers through MPMH in the MRMC situation, the potential of spatial reuse is released.

The main contributions of this paper are threefold. Firstly, we formulate MPMH-MRMC scheduling problem into a mixed-integer linear programming (MILP) to accommodate the traffic of all flows within a minimum number of time slots. The signal to interference plus noise ratio (SINR) constraint is considered in the MILP. Secondly, a low-computation heuristic scheduling algorithm is proposed to solve the MILP, which is NP-hard. The results of the algorithm are close to the optimal solution. Finally, extensive simulation results are provided to verify the superior performance in terms of delay and throughput compared with existing works.

The rest of this paper is organized as follows. Related works are presented in Section 2. We describe the system overview including directional MAC structure, transmission model, interference level, and problem overview in Section 3. In Section 4, the optimal MPMH-MRMC problem is formulated as an MILP. In Section 5, a lowcomputation heuristic scheduling algorithm is proposed to solve the MILP. In Section 6, we evaluate the performance of MPMH-MRMC under various traffic patterns and simulation parameters. This paper is summarized in Section 7.

\section{Related work}

There has been considerable work on transmission scheduling for mmWave communications in the literatures. As time division multiple access (TDMA) is adopted in the standards for $60 \mathrm{GHz}$ WPANs [1], i.e., ECMA-387 and IEEE 802.15.3c, some schemes were proposed based on TDMA [3,10-14]. Motivated by the fact that mmWave can attain high space utilization with highly directional point-to-point communications, many concurrent transmission algorithms were presented. These algorithms can be classified into two categories, namely, conflict graph-based and SINR-based scheduling schemes.

In the conflict graph-based scheduling schemes, maximum independent set-based scheduling was proposed $[5,8,15]$. In these protocols, interference was modeled as a conflict graph, where the interference between the links in an independent set is zero. The interference model is constructed on the assumption that zero beamwidth [15] or ideal antenna gain, i.e., constant antenna gain within the beamwidth and zero gain outside the beamwidth $[5,8]$.

In the SINR-based scheduling schemes, considering the sidelobe antenna gain, the concept of exclusive region was introduced to enable concurrent transmissions $[12,16,17]$. When the exclusive region conditions are satisfied, multiple links transmitting simultaneously always outperforms TDMA scheme. Furthermore, J. Qiao et al. proposed a concurrent transmission scheduling algorithm for indoor IEEE 802.15.3c WPANs [3]. When MUI is below a specific threshold, multiple links are scheduled to transmit concurrently to maximize the number of flows scheduled in the network such that the QoS requirement of each flow is satisfied.

Since each node in WPANs can reach each other without blockage, considerable work has been put forward to settle the single hop concurrent scheduling problem $[3,4,6,8,18,19]$. To address the link outage problem caused by blockage and combat severe attenuation to improve network throughput, multi-hop concurrent transmission (MHCT) scheme was proposed [5, 20]. The MHCT scheme concurrently transmits the traffic of flows through multiple hops without causing harmful interference. Y. Niu et al. proposed an MPMH scheduling scheme in [1], where the traffic across links of low channel quality is transmitted through MPMH to enhance spatial reuse. However, the potential of MPMH is not fully boosted in the SRSC situation. L. Zhou et al. exploited multiple radio interfaces over multiple channels in [8] to enhance the performance. Nevertheless, it is applied in the single hop scene employing conflict graph. To the best of our knowledge, the previous works in mmWave WPANs employed MPMH or MRMC for data transmission. Taking into account the unique features of mmWave WPANs (such as line of sight (LOS) transmission, high propagation loss, and directivity), it is likely that employing $\mathrm{MPMH}$ and MRMC jointly in mmWave WPANs can improve the network throughput and transmission delay. 


\section{System overview}

\subsection{Directional MAC structure}

An indoor IEEE 802.15.3c WPAN consisting of $N-1$ wireless nodes (WNs) and a piconet controller (PNC) is considered. As an example, a 6-node WPAN is illustrated in Fig. 1. Each WN and PNC in the network are equipped with electronically steerable directional antennas for directional data transmissions by beamforming. The superframe contains three periods. Network synchronization and control messages broadcasting from the PNC are processed in the beacon period (BP). The transmission requests of the WNs are sent to the PNC in the contention access period (CAP). The data transmission among WNs in peer-to-peer fashion occurs during the channel time allocation period (CTAP) [3]. The number of timeslots involved in the CTAP during each superframe is adjusted adaptively according to the total occupied time slots. Besides, the system runs a bootstrapping program [1], through which the up-to-date network topology and the location information of other devices are known by each device.

\subsection{Transmission model}

\subsubsection{Antenna model}

The directional antenna model proposed in [4] has been widely used, which has a main lobe of Gaussian form and constant level of side lobes. In this model, the directional antenna gain expressed in units of $\mathrm{dB}$ is defined as $G\left(\alpha, \theta_{-3 \mathrm{~dB}}\right)$, where $\alpha$ is the angle between the transmission direction (receiving direction) and center line of the transmitter's (receiver's) sector beam, $\theta_{-3 \mathrm{~dB}}$ is the antenna's half-power bandwidth (HPBW).

\subsubsection{Channel model}

In this paper, directional LOS transmission is adopted to achieve high data rate transmission and maximize

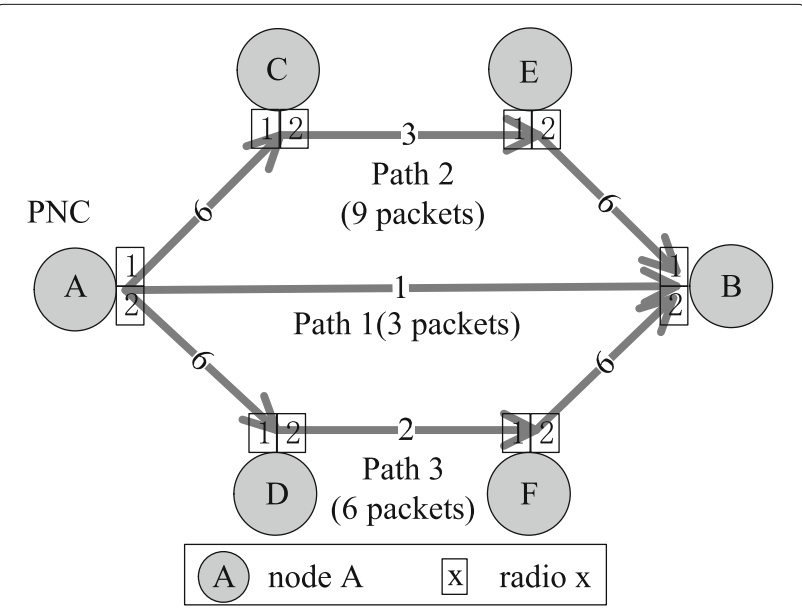

Fig. 1 Transmission path the power efficiency [1]. For each directional link $l_{i}$, we denote its transmitter and receiver nodes as $t_{i}$ and $r_{i}$, respectively. We also denote the distance in meters between $t_{i}$ and $r_{i}$ as $d_{i i}$. For example, we define the link from $A$ to $B$ in Fig. 1 as $l_{2}$, then $t_{2}$ and $r_{2}$ represent node $A$ and $B$, respectively. The distance between node $A$ and $B$ is given by $d_{22}$. Considering the low user mobility and LOS transmission for indoor WPANs, the path loss at distance $d_{i i}$ in $\mathrm{dB}$ can be modeled as

$$
L\left(d_{i i}\right)=A_{\mathrm{LOS}}+20 \log 10(f)+10 \rho \log 10\left(d_{i i}\right),
$$

where $f$ is the carrier frequency in $\mathrm{GHz}, \rho$ is the path loss exponent, and $A_{\mathrm{LOS}}$ is a constant. The values of $\rho$ and $A_{\mathrm{LOS}}$ depend on the specific scenario [4]. As the average value of the instantaneous path loss possesses low deviations, the assumption that no shadowing fading in $A_{\mathrm{LOS}}$ is reasonable. The received signal power of $r_{i}$ in $\mathrm{dB}$ is given by

$$
P_{r}(i, i)=P_{t}\left(t_{i}\right)+G_{t_{i}}+G_{r_{i}}-L\left(d_{i i}\right),
$$

where $P_{t}\left(t_{i}\right)$ is the transmit power of $t_{i}, G_{t_{i}}$ is the transmit antenna gain of $t_{i}$, and $G_{r_{i}}$ is the receive antenna gain of $r_{i}$. Similarly, if link $l_{i}$ and $l_{j}$ transmit with the same frequency simultaneously, the received interference power from $t_{j}$ to $r_{i}$ can be calculated as

$$
P_{r}(i, j)=P_{t}\left(t_{j}\right)+G_{t_{j}}+G_{r_{i}}-L\left(d_{i j}\right) .
$$

\subsection{Interference level}

For each link $l_{i}$, we suppose the minimum SINR to support its transmission rate $c_{i}$ as $\operatorname{MS}\left(c_{i}\right)$. Therefore, concurrent transmissions are supported if the SINR of each link $l_{i}$ is not smaller than $\mathrm{MS}\left(c_{i}\right)$ [4]. To guarantee the successful transmission of link $l_{i}$, the SINR constraint can be expressed as

$$
\frac{P_{r}(i, i)}{P_{N}+\sum_{j \neq i} P_{r}(i, j)} \geq \operatorname{MS}\left(c_{i}\right),
$$

where $P_{r}(i, j)$ denotes the interference power from link $l_{j}$, and $l_{j}$ denotes the link concurrent transmitted with link $l_{i}$ at the same frequency. $P_{N}=N_{0} W_{0}$ is the ambient noise power level, where $W_{0}$ is the bandwidth and $N_{0}$ is the onesided power spectra density of white Gaussian noise. (4) can also be written as

$$
\frac{\operatorname{MS}\left(C_{i}\right) \cdot \sum_{j \neq i} P_{r}(i, j)}{P_{r}(i, i)-\operatorname{MS}\left(C_{i}\right) \cdot P_{N}} \leq 1 .
$$

To facilitate the analysis, interference level is defined to quantize the interference between any two concurrent transmitted links at the same frequency. Then the interference level caused by link $l_{j}$ to $l_{i}$ can be defined as

$$
W_{i, j}=\frac{\operatorname{MS}\left(C_{i}\right) \cdot P_{r}(i, j)}{P_{r}(i, i)-\operatorname{MS}\left(C_{i}\right) \cdot P_{N}} .
$$


(5) is satisfied when $\sum_{j \neq i} W_{i, j} \leq 1$. Therefore, the SINR condition of a successful transmission of link $l_{i}$ can be summarized as

$$
I_{i}=\sum_{j \neq i} W_{i, j} \in[0,1]
$$

where $l_{j}$ denotes the link concurrent transmitted with link $l_{i}$ at the same frequency. We define $I_{i}$ as the cumulative interference level of link $l_{i}$. With the definition of cumulative interference level, the SINR condition is converted to a summation form.

For link $l_{i}$, each radio interface of transmitter $t_{i}$ and receiver $r_{i}$ is equipped with a directional antenna that can be tuned to a specific direction and channel for data transmission. We denote the set of radios of node $t_{i}$ and $r_{i}$ as $\mathbf{R}_{t_{i}}$ and $\mathbf{R}_{r_{i}}$. We also define the set of channels as $\mathbf{C}$. During the scheduling, we should specify the radio that node $t_{i}$ employs, the radio that node $r_{i}$ uses, and the channel occupied by link $l_{i}$. The definition of tuple link is proposed for this purpose. The link $l_{i}$ between $t_{i}$ and $r_{i}$ can be further defined as a node-radio-channel tuple, i.e., tuple link. The set of tuple links of link $l_{i}$ is defined as $\mathbf{L}_{i}$. According to the method of permutation and combination, the number of tuple links of link $l_{i}$ is given by $\left|\mathbf{L}_{i}\right|=\left|\mathbf{R}_{t_{i}}\right| \cdot\left|\mathbf{R}_{r_{i}}\right| \cdot|\mathbf{C}|$. The $x$-th tuple link of link $l_{i}$ is expressed as $l_{i}^{x}$. For example, the link from node $A$ to node $B$ in Fig. 1 is denoted as $l_{2}$. Assuming that $\mathbf{R}_{A}=\left\{R_{A, 1}, R_{A, 2}\right\}, \mathbf{R}_{B}=\left\{R_{B, 1}, R_{B, 2}\right\}$, and $\mathbf{C}=\left\{C_{1}, C_{2}\right\}$. The tuple link set of link $l_{2}$ is given by $\mathbf{L}_{2}=\left\{\left(R_{A, z_{1}}, R_{B, z_{2}}, C_{z_{3}}\right), z_{1}, z_{2}, z_{3}=1,2\right\}$, which has $\left|\mathbf{L}_{2}\right|=8$ tuple links. Tuple link $\left(R_{A, 1}, R_{B, 2}, C_{1}\right)$ means that there is a link from node $A$ to $B$, node $A$ employs radio 1 , node $B$ employs radio 2 , and channel 1 is occupied by the link.

The interference level caused by tuple $\operatorname{link} l_{j}^{y}$ to $l_{i}^{x}$ can be defined as

$$
W_{i, j}^{x, y}=\left\{\begin{array}{ll}
0, & \text { case } 1 \text { or } 2 \text { or } 3 \\
\infty, & \text { case } 4 \text { or } 5 \\
W_{i, j}, & \text { case } 6
\end{array} .\right.
$$

The cases are defined as follows:

- Case 1: $l_{i}^{x}$ does not exist in reality, i.e., $t_{i}=r_{i}$.

- Case 2: $l_{i}^{x}=l_{j}^{y}$, which means that $i=j, x=y \cdot l_{i}^{x}$ and $l_{j}^{y}$ are the same tuple link.

- Case 3: $l_{i}^{x} \neq l_{j}^{y}$, which means that $l_{i}^{x}$ and $l_{j}^{y}$ are different tuple links. They operate on a diverse channel with disparate radios.

- Case 4: $l_{i}^{x} \neq l_{j}^{y}$, they share the same radio.

- Case 5: $l_{i}^{x} \neq l_{j}^{y}$, they have the common node(s) and operate on the same channel with disparate radios. The common node(s) transmit and receive simultaneously [15].
- Case 6: Except for the above cases, we obtain the interference level $W_{i, j}$ with (6).

\subsection{Problem overview}

To improve the throughput of the network, the flow with low transmission rate on the direct link will be transmitted through multiple paths to enhance the transmission efficiency. The object of the schedule is to clear all the flows within a minimum number of time slots.

As an example, a 6-node WPAN, i.e., $A, B, C, D, E$, and $F$, is illustrated in Fig. 1 to describe the basic idea of MPMH-MRMC. The numbers in the middle of the direct links indicate the number of packets can be transmitted by these links in one time slot. The flow from $A$ to $B$ consists of 18 packets. In the MPMH-MRMC, three paths, the path of $A \rightarrow B$ (path 1), $A \rightarrow C \rightarrow E \rightarrow B$ (path 2), and $A \rightarrow D \rightarrow F \rightarrow B$ (path 3) are selected for the flow from $A$ to $B$. The traffic is proportionally distributed according to the lowest transmission rate on each path [1]. Therefore, three packets, nine packets, and six packets are distributed to paths 1,2 , and 3, respectively. Assuming that each node has two radios and there are two channels, the scheduling result of MPMH-MRMC is illustrated in Fig. 2, where the unit of the scale is time slot. The concurrent transmissions in the same time slots are named pairing in this paper. It is shown that the schedule has 5 pairings, which means that the flow can be scheduled in 5 time slots. In the 4-th pairing, tuple links $\left(R_{A, 1}, R_{B, 1}, C_{1}\right),\left(R_{B, 2}, R_{F, 2}, C_{2}\right),\left(R_{C, 1}, R_{E, 1}, C_{1}\right)$, and $\left(R_{C, 2}, R_{E, 2}, C_{2}\right)$ transmit concurrently. However, 9 time slots are needed if they are transmitted from $A$ to $B$ directly. From the results, it is known that the efficiency of transmission is improved.

\section{Problem formulation}

In this section, we convert the problem of optimal MPMH-MRMC scheduling into a standard MILP based on the Reformulation-Linearization Technique (RLT) [21]. We list the notations in Table 1 for better understanding of the problem formulation.

\subsection{Problem formulation and analysis}

We assume that there are $V$ flows to be scheduled by the PNC. For flows with low transmission rates or high traffic demands, more time slots are allocated to handle their traffic demands. Since these extra time slots cannot be used in concurrent transmissions, the system performance will deteriorate. To improve throughput, they should be transmitted through multiple paths to enhance spatial reuse. The flow transmitted through multiple paths will be selected with the method in [1].

The number of paths of the $v$-th flow is denoted as $M_{\nu}$. We indicate the number of hops of the $p$-th path of the $v$-th flow as $H_{v p}$. For the $v$-th flow, we denote its traffic 


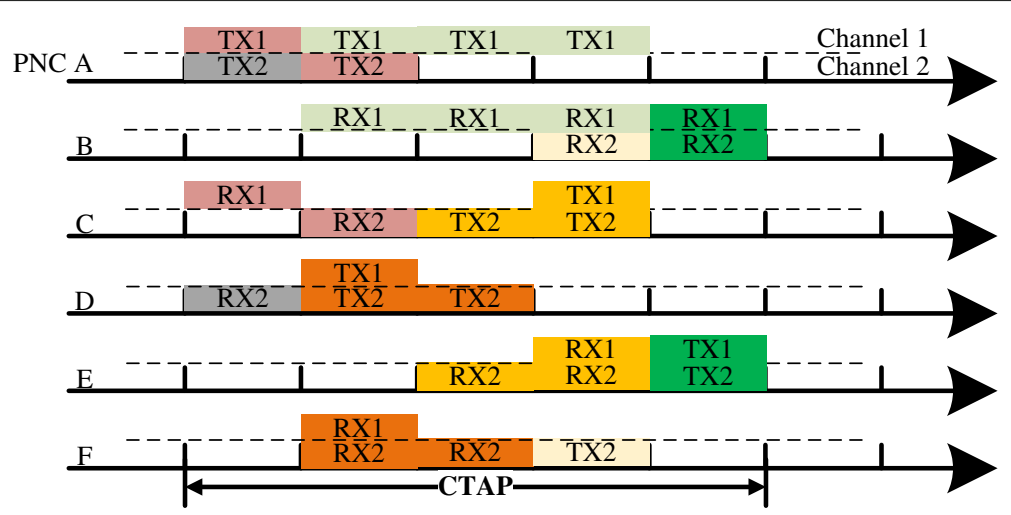

Fig. 2 An example of MPMH-MRMC operation applying MILP

demand and the traffic demand distributed to the $p$-th path as $d_{v}$ and $d_{v p}$, respectively. The links of a path are sorted by the links' hop number, i.e., the link of the first hop of the $p$-th path is denoted as the first link of the $p$ th path. The $i$-th link of the $p$-th path of the $v$-th flow is defined as $l_{v p i}$, and its transmission rate is $c_{v p i}$. According to the previous definition, there exists $\left|\mathbf{L}_{v p i}\right|$ tuple links between transmitter $t_{v p i}$ and receiver $r_{v p i}$. Their transmission rates are also $c_{v p i}$. We assume that there are $K$ pairings in the schedule to handle all the traffics, and the $k$-th pairing consists one time slot. We also use indicator function $a_{v p i}^{k}, a_{v p i}^{x k}$ to express whether link $l_{v p i}$, tuple

Table 1 Notation in problem formulation

\begin{tabular}{ll}
\hline Symbol & Description \\
\hline$K$ & The number of pairings in a schedule \\
$V$ & The number of flows in the network \\
$M_{v}$ & The number of paths of the $v$-th flow \\
$H_{v p}$ & The number of hops of the $p$-th path of the $v$-th flow \\
$d_{v}$ & The traffic demand of the $v$-th flow \\
$d_{v p}$ & The traffic demand distributed to the $p$-th path \\
$I_{v p i}$ & The $i$-th link of the $p$-th path of the $v$-th flow \\
$c_{v p i}$ & The transmission rate of link $I_{v p i}$ \\
$\mathbf{L}_{u q j}$ & The set of tuple links of link $I_{v p i}$ \\
$I_{v p i}^{x}$ & The $x$-th tuple link of link $I_{v p i}$ \\
$t_{v p i}$ & The transmitter of link $I_{v p i}$ (tuple link $I_{v p i}^{x}$ ) \\
$r_{v p i}$ & The receiver of link $I_{v p i}$ (tuple link $\left.\left.\right|_{v p i} ^{x}\right)$ \\
$a_{v p i}^{k}$ & A binary variable to indicate whether link $I_{v p i}$ is scheduled in \\
$a_{v p i}^{x k}$ & the $k$-th pairing \\
$\mathbf{R}_{t_{p i}}$ & A binary variable to indicate whether tuple link $\left.\right|_{v p i} ^{x}$ is scheduled \\
$\mathbf{C}$ & in the $k$-th pairing \\
$W_{v p i, u q j}^{x, y}$ & The set of radios of node $t_{v p i}$ \\
\hline & The set of channels in the network
\end{tabular}

link $l_{v p i}^{x}$, i.e., the $x$-th tuple link of link $l_{v p i}$, is scheduled in the $k$-th pairing, respectively. $a_{v p i}^{k} / a_{v p i}^{x k}$ is assigned 1 when $l_{v p i} / l_{v p i}^{x}$ is scheduled. Besides, the interference level caused by tuple link $l_{u q j}^{y}$ to $l_{v p i}^{x}$ is defined as $W_{v p i, u q j}^{x, y}$.

For example, we assume the flow from node $A$ to $B$ in Fig. 1 is the first flow, then we have $M_{1}=3, H_{11}=1$, $H_{12}=3$, and $H_{13}=3$. Besides, the traffic demand of the first flow and the traffic demand distributed to the three paths are given by $d_{1}=18, d_{11}=3, d_{12}=9$, and $d_{13}=6$. The link from $A$ to $B$ is the first link of the first path of the first flow. It is denoted as $l_{111}$, and the rate of link $l_{111}$ is $c_{111}$. Similarly, the link from $D$ to $F$ is given by $l_{132}$. Then, the transmitter $t_{111}$ and receiver $r_{111}$ represent node $A$ and $B$, respectively. The number of tuple links between $A$ and $B$ is $\left|\mathbf{L}_{111}\right|$. Besides, $a_{111}^{2}$ and $a_{111}^{32}$ are indicators to express whether link $l_{111}$ and tuple link $l_{111}^{3}$, i.e., the third tuple link of link $l_{111}$ are scheduled in the second pairing. The interference level caused by tuple $\operatorname{link} l_{132}^{2}$ to $l_{111}^{3}$ is defined as $W_{111,132}^{3,2}$.

An optimal schedule should clear all the flows within a minimum number of time slots, which implies maximum parallelism of transmissions. We formulate the optimal MPMH-MRMC scheduling problem P1 as:

$$
\text { P1: } \min _{a_{v p i}^{x k}} K=f u n\left(a_{v p i}^{x k}, d_{v p}, c_{v p i}, W_{v p i, u q j}^{x, y}\right)
$$

s.t.

$$
\begin{aligned}
& \sum_{p=1}^{M v} d_{v p}=d_{v}, \forall v \\
& a_{v p i}^{k} \in\left\{\begin{array}{ll}
\{0,1\}, & \text { if } d_{v p}>0 \& i \leq H_{v p} \\
0\}, & \text { otherwise }
\end{array}, \forall v, p, i, k\right. \\
& \sum_{k=1}^{K} a_{v p i}^{k} \begin{cases}\leq\left\lceil\frac{d_{v p}}{c_{v p i}}\right], & \text { if } d_{v p}>0 \& i \leq H_{v p}, \forall v, p, i \\
=0, & \text { otherwise }\end{cases}
\end{aligned}
$$




$$
\begin{gathered}
a_{v p i}^{x k} \in\left\{\begin{array}{ll}
\{0,1\}, & \text { if } a_{v p i}^{k}=1 \\
\{0\}, \quad \text { otherwise }
\end{array}, \forall v, p, i, k, x\right. \\
a_{v p i}^{k} \leq \sum_{x=1}^{\left|\mathbf{L}_{v p i}\right|} a_{v p i}^{x k} \leq a_{v p i}^{k} \min \left\{\left|\mathbf{R}_{t_{v p i}}\right|,\left|\mathbf{R}_{r_{v p i} \mid}\right|,|\mathbf{C}|\right\}, \quad \forall v, p, i, k \\
\sum_{k=1}^{K} \sum_{x=1}^{\left|\mathbf{L}_{v p i}\right|} a_{v p i}^{x k}\left\{\begin{array}{l}
=\left\lceil\frac{d_{v p}}{c_{v p i}}\right. \\
=0, \text { otherwise; }
\end{array}, \text { if } d_{v p}>0 \& i \leq H_{v p} \quad, \quad \forall v, p, i\right.
\end{gathered}
$$

$\sum_{k=1}^{K^{*}} \sum_{x=1}^{\left|\mathbf{L}_{p p i}\right|} a_{v p i}^{x k} /\left\lceil\frac{d_{v p}}{c_{v p i}}\right\rceil \geq a_{v p(i+1)}^{K^{*}+1}$, if $H_{v p}>1, \forall v, p, i \leq H_{v p}-1, K^{*} \leq K$

$$
a_{v p i}^{x k}+a_{u q j}^{y k} \leq 1, \text { if } W_{v p i, u q j}^{x, y}>1, \forall v, p, i, x, u, q, j, y, k
$$

$$
\sum_{u=1}^{V} \sum_{q=1}^{M_{u}} \sum_{j=1}^{H_{u q}} \sum_{y=1}^{\mathbf{L}_{u q j} \mid} a_{v p i}^{x k} a_{u q j}^{y k} W_{v p i, u q j}^{x, y} \leq 1, \forall v, p, i, x, k .
$$

This is an MINLP problem, which is generally NP-hard. The explanations of these constraints are as follows:

- Constraint (10) indicates regular flow restriction.

- Constraints (11) and (12) indicate that link $l_{v p i}$ should be scheduled at most $\left\lceil d_{v p} / c_{v p i}\right\rceil$ times in the $K$ pairings.

- Constraints (13) and (14) indicate that at most $\min \left\{\left|\mathbf{R}_{t_{v p i}}\right|,\left|\mathbf{R}_{r_{v p i}}\right|,|\mathbf{C}|\right\}$ tuple links between two nodes $t_{v p i}, r_{v p i}$ could be scheduled in one pairing.

- Constraint (15) indicates that the traffic of link $l_{v p i}$ should be accommodated in the schedule.

- Constraints (16)-(18) represent scheduling restrictions. Constraint (16) indicates the inherent order of transmission in each path, the $(i+1)$-th link of the $p$-th path of the $v$-th flow should be scheduled after the $i$-th link. Constraints (17)-(18) indicate SINR restriction for concurrent transmissions.

\subsection{Problem reformulation}

Problem P1 has a nonlinear constraint (18). We apply the RLT to obtain a linear relaxation [21]. Problem P1 will become a standard MILP, which can be solved by some existing sophisticated algorithms, such as the branch-andbound method.

For constraint (18), we define a substitution variable $\mu_{v p i, u q j}^{x, y, k}=a_{v p i}^{x k} a_{u q j}^{y k}$. We have $0 \leq a_{v p i}^{x k} \leq 1$ and $0 \leq a_{u q j}^{y k} \leq$
1. Then, we can obtain the RLT bound-factor product constraints for $\mu_{v p i, u q j}^{x y k}$ as

$$
\left\{\begin{array}{l}
\left\{\begin{array}{l}
\left.\left[a_{v p i}^{x k}-0\right] \cdot\left[a_{u q j}^{y k}-0\right]\right\}_{L S} \geq 0 \\
{\left[1-a_{v p i}^{x k}\right] \cdot\left[a_{u q j}^{y k}-0\right]}
\end{array}\right\}_{L S} \geq 0 \\
\left\{\left[a_{v p i}^{x k}-0\right] \cdot\left[1-a_{u q j}^{y k}\right]\right\}_{L S} \geq 0 \\
\left\{\left[1-a_{v p i}^{x k}\right] \cdot\left[1-a_{u q j}^{y k}\right]\right\}_{L S} \geq 0
\end{array}, \forall v, p, i, x, y, k\right.
$$

where $\{\cdot\}_{\mathrm{LS}}$ represents a linearization step under $\mu_{v p i, u q j}^{x, y, k}=$ $a_{v p i}^{x k} a_{u q j}^{y k}$. By substituting $\mu_{v p i, u q j}^{x, y, k}=a_{v p i}^{x k} a_{u q j}^{y k}$, we obtain

$$
\left\{\begin{array}{l}
\mu_{v p i, u q j}^{x, y, k} \geq 0 \\
a_{v p i}^{x k}-\mu_{v v i, u q j}^{x, y, k} \geq 0 \\
a_{u q j}^{y k}-\mu_{v p i, u q j}^{x, y, k} \geq 0 \\
1-a_{v p i}^{x k}-a_{u q j}^{y k}+\mu_{v p i, u q j}^{x, y, k} \geq 0
\end{array}, \forall v, p, i, x, y, k .\right.
$$

Substituting $\mu_{v p i, u q j}^{x y k}$ into constraint (18), we obtain an MILP relaxation P2 as

$$
\text { P2: } \min _{a_{v p i}^{x k}} K=f u n\left(a_{v p i}^{x k}, \mu_{v p i, u q j}^{x, y, k}, d_{v p}, c_{v p i}, W_{v p i, u q j}^{x, y}\right)
$$

\section{Subject to}

$$
\sum_{u=1}^{V} \sum_{q=1}^{M_{u}} \sum_{j=1}^{H_{u q}} \sum_{y=1}^{\left|\mathbf{L}_{u q j}\right|} \mu_{v p i, u q j}^{x, y, k} W_{v p i, u q j}^{x, y} \leq 1, \forall v, p, i, x, k .
$$

$$
\text { (10)-(17), (20). }
$$

\subsection{Example}

As an example, a 6-node WPAN is illustrated in Fig. 1. Without loss of generality, the interference level between tuple link $l_{v p i}^{x}$ and $l_{u q j}^{y}$, i.e., $W_{v p i, u q j}^{x, y}$, of case 6 is assumed to be 0.1 in Fig. 1. As mentioned before, the schedule contains five time slots in the 2-radio 2-channel situation and the scheduling process is illustrated in Fig. 2. However, the transmission phase of the schedule consists of 18 time slots when using the SPSH-SRSC method. It consumes nine time slots when applying the MPMH method [1]. MPMH-MRMC reduces the time slots for transmission by 72 and $44 \%$ compared with the SPSH-SRSC and MPMH methods. However, the reformulated MILP P2 is NP-hard. Applying an open-source MILP solver, lp solver ver 5.5.2.0 [2] to obtain the optimal solution will take significantly long computation time, e.g., several minutes. It is not practical for mmWave WPANs where the duration of a time slot is only several $\mu \mathrm{s}$. In the next section, we propose a low-computation heuristic algorithm. 


\section{The MPMH-MRMC scheme}

To initialize problem P2, the suitable transmission paths should be selected. Besides, the traffic of each flow should be distributed to its selected paths efficiently. The path selection and traffic distribution method proposed in [1] are adopted. In this section, we demonstrate a heuristic scheduling algorithm that can solve problem P2 with low computational complexity. The notations are listed in Table 2 to facilitate the understanding of MPMH-MRMC.

\subsection{Symbols in MPMH-MRMC scheme}

Assuming that the number of radios of each node in the network is the same, which is denoted as $R_{0}$. The number of channels $|\boldsymbol{C}|$ is no less than $R_{0}$. In a pairing, the SINR of each tuple link should be larger than its threshold. It means that the maximum number of tuple links in the same pairing is $R_{0} \cdot\lfloor N / 2\rfloor$. According to the traffic of each path, the number of time slots for a link to accommodate its traffic is set as the weight of this link.

In the transmission scheduling, the set of selected paths of all flows is defined as $\mathbf{P}_{S}$. The set of links in $\mathbf{P}_{s}$ is denoted as $\mathbf{H}$. As mentioned in Section 4.2, the links of a path are sorted by the links' hop number. We denote the $i$-th link of the $p$-th path of $\mathbf{P}_{s}$ as $l_{p i}$. The $x$-th tuple link of link $l_{p i}$ is given by $l_{p i}^{x}$. The initial weight of each link $l_{p i}$ is defined as $w_{p i}$. The remaining weight of link $l_{p i}$ in the $t$-th pairing is defined as $w_{p i}^{t}$. The $t$-th pairing consists of at least

\section{Table 2 Notation in MPMH-MRMC}

\begin{tabular}{|c|c|}
\hline Symbol & Description \\
\hline$R_{0}$ & The number of radios of a node in the network \\
\hline$N$ & The number of nodes \\
\hline $\mathbf{P}_{S}$ & Set of selected paths of all flows \\
\hline $\mathbf{H}$ & Set of links in $\mathbf{P}_{5}$ \\
\hline Ipi & The $i$-th link of the $p$-th path \\
\hline$\left.\right|_{p i} ^{x}$ & The $x$-th tuple link of link $/_{p i}$ \\
\hline$W_{p i}$ & The initial weight of link $I_{p i}$ \\
\hline$W_{p i}^{t}$ & The remaining weight of link $I_{p i}$ in the $t$-th pairing \\
\hline$t_{p i}$ & The transmitter of link $I_{p i}\left(\right.$ tuple link $\left.\left.\right|_{p i} ^{x}\right)$ \\
\hline$r_{p i}$ & The receiver of link $/_{p i}\left(\right.$ tuple link $\left.\left.\right|_{p i} ^{x}\right)$ \\
\hline $\mathbf{R}_{t_{p i}}^{t}$ & Set of unused radios of transmitter $t_{p i}$ in the $t$-th pairing \\
\hline $\mathbf{R}_{r_{p i}}^{t}$ & Set of unused radios of receiver $r_{p i}$ in the $t$-th pairing \\
\hline$F_{p}$ & The hop number of the first unscheduled link of the $p$-th path \\
\hline $\mathbf{P}_{m u h}^{t}$ & $\begin{array}{l}\text { Set of unvisited paths with the largest number of unscheduled } \\
\text { links }\end{array}$ \\
\hline $\mathbf{P}_{u}^{t}$ & Set of unvisited paths in the $t$-th pairing \\
\hline $\mathbf{H}^{t}$ & Set of links in the $t$-th pairing \\
\hline $\mathbf{H}_{0}^{t}$ & Set of tuple links in the $t$-th pairing \\
\hline$\delta^{t}$ & Number of time slots of the $t$-th pairing \\
\hline$P_{p i}^{x}$ & The cumulative interference level of tuple link $\left.\right|_{p i} ^{x}$ \\
\hline
\end{tabular}

one time slot. The transmitter and receiver of link $l_{p i} /$ tuple link $l_{p i}^{x}$ are denoted as $t_{p i}$ and $r_{p i}$, respectively. The sets of unoccupied radios of nodes $t_{p i}$ and $r_{p i}$ in the $t$-th pairing are expressed as $\mathbf{R}_{t_{p i}}^{t}$ and $\mathbf{R}_{r_{p i}}^{t}$. Let $F_{p}$ denote the hop number of first unscheduled link of the $p$-th path. The set of unvisited paths with the largest number of unscheduled hops in the $t$-th pairing is given by $\mathbf{P}_{m u h}^{t}$. The set of paths that are not visited in the $t$-th pairing is denoted as $\mathbf{P}_{u}^{t}$. The sets of links and tuple links of the $t$-th pairing are defined as $\mathbf{H}^{t}$ and $\mathbf{H}_{0}^{t}$, respectively. Besides, we denote the number of time slots of the $t$-th pairing as $\delta^{t}$.

For example, the network illustrated in Fig. 1 has one flow from $A$ to $B$. Then, $\mathbf{P}_{S}$ contains three paths: $A \rightarrow B$ (first path, $p=1$ ), $A \rightarrow C \rightarrow E \rightarrow B$ (second path, $p=2$ ), and $A \rightarrow D \rightarrow F \rightarrow B$ (third path, $p=3$ ). Thus, $\mathbf{H}$ consists of seven links. The first link of the third path is $A \rightarrow D$, which is denoted by $l_{31}$. The initial weight of link $l_{31}$ is $w_{31}=1$. The remaining weight of link $l_{31}$ in the $t$-th pairing is defined as $w_{31}^{t}$. The transmitter $t_{31}$ and receiver $t_{31}$ of link $l_{31}$ represent nodes $A$ and $B$, respectively. Initially, we have $F_{p}=1, p=1,2,3$, which means that the first link of the three paths has not been scheduled. Since path 2 and path 3 both contain 3 links before the schedule, $\mathbf{P}_{\text {muh }}^{1}$ contains 2 paths: the second and third path. We have $\mathbf{P}_{\text {muh }}^{1}=\{p \mid p=2,3\}$.

\subsection{Working mechanism of MPMH-MRMC scheme}

In the MPMH-MRMC scheme, we define two mechanisms, namely, the link selection mechanism and the tuple link schedule mechanism.

Link selection mechanism: Assuming that the set of unvisited paths with the largest number of unscheduled hops in the $t$-th pairing is denoted by $\mathbf{P}_{m u h}^{t}$. As mentioned in 5.1, the hop number of the first unscheduled link of the $p$-th path is defined as $F_{p}$. The weight of $\operatorname{link} l_{p F_{p}}, p \in \mathbf{P}_{\text {muh }}^{t}$ in the $t$-th pairing is denoted as $w_{p F_{p}}^{t}$. The link with minimum weight $w_{p F_{p}}^{t}, p \in \mathbf{P}_{m u h}^{t}$ will be selected. We have

$$
l_{p i} \triangleq \min _{p \in \mathbf{P}_{m u h}^{t}} w_{p F_{p}}^{t} .
$$

Tuple link schedule mechanism: Assuming that link $l_{p i}$ is selected in the $t$-th pairing. The tuple link $l_{p i}^{x_{0}}$ brings minimum interference to the concurrent transmissions in $\mathbf{H}_{0}^{t}$ will be selected. We denote the cumulative interference level of tuple link $l_{p i}^{x}$ as $I_{p i}^{x}$. Then, the tuple link selected is given by

$$
l_{p i}^{x_{0}} \triangleq \min _{I_{q j}^{y}} \max \left\{I_{q j}^{y} \mid l_{p i}^{x} \notin \mathbf{H}_{0}^{t}, l_{q j}^{y} \in \mathbf{H}_{0}^{t} \cup\left\{l_{p i}^{x}\right\}\right\} .
$$

The maximum cumulative interference level of a tuple link in $\mathbf{H}_{0}^{t} \cup\left\{l_{p i}^{x_{0}}\right\}$ is denoted as $I_{0}$. We have

$$
I_{0}=\max \left\{I_{q j}^{y} \mid l_{q j}^{y} \in \mathbf{H}_{0}^{t} \cup\left\{l_{p i}^{x_{0}}\right\}\right\} .
$$


If $I_{0} \leq 1$, tuple link $l_{p i}^{x_{0}}$ will be added to $\mathbf{H}_{0}^{t}$ and scheduled. Link $l_{p i}$ will be added to $\mathbf{H}^{t}$ accordingly.

Considering the SINR constraint, the flow chart of the heuristic traffic scheduling algorithm is presented in Fig. 3. The algorithm can be described as

Step 1: Select the tuple links to schedule in a pairing. Select link $l_{p i}$ according to the link selection mechanism until $\mathbf{P}_{u}^{t}=\phi$ or $\left|\mathbf{H}^{t}\right|>R_{0} \cdot\lfloor N / 2\rfloor$. The corresponding tuple link $l_{p i}^{x}$ is scheduled according to the tuple link schedule mechanism;

Step 2: Schedule the unoccupied radios of selected link $l_{p i}$ in $\mathbf{H}^{t}$. The corresponding tuple link $l_{p i}^{x}$ is scheduled according to the tuple link schedule mechanism;

Step 3: Determine the number of time slots of the pairing. Repeat step 2 until no more tuple links can be added to $\mathbf{H}_{0}^{t}$. The minimum time slots needed for a link $l_{p i}$ in $\mathbf{H}^{t}$ to accommodate its traffic is $\delta_{p i}^{t}$. The number of time slots of the $t$-th pairing is $\delta^{t}=\min _{l_{p i} \in \mathbf{H}^{t}}\left\{\delta_{p i}^{t}\right\}$;

Step 4: Update $\mathbf{H}, F_{p}$, and the weight of scheduled links. Go to step 1 if $|\mathbf{H}|>0$, end the algorithm when $|\mathbf{H}| \leq 0$.

The pseudo code of the heuristic traffic scheduling algorithm is presented in Algorithm 1. Line 4 obtains the suitable link $l_{p i}$. Firstly, it gets the set of unvisited paths with the largest number of unscheduled hops $\mathbf{P}_{m u h}^{t}$ in the $t$-th pairing. Then, it gets the link of path $p \in \mathbf{P}_{m u h}^{t}$ with the minimum weight $w_{p F_{p}}^{t}$, which is denoted as $l_{p i}$. Line 5 selects the tuple link $l_{p i}^{x}$ that brings minimum interference to the tuple links in $\mathbf{H}_{0}^{t}$. Lines 8-12 utilize the idle radios of the transmitters and receivers of scheduled links in $\mathbf{H}^{t}$ when there are remained data to transmit. Line 13 obtains the duration of $t$-th pairing $\delta^{t}$. $\delta^{t}$ is the minimum time needed for a link in $\mathbf{H}^{t}$ to accommodate its traffic. Lines 14-17 update the weight of each scheduled link and

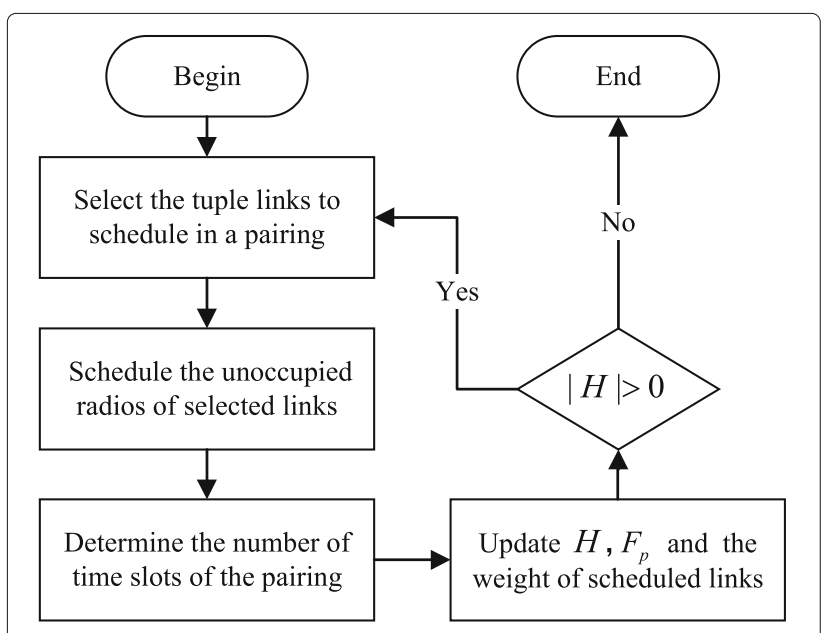

Fig. 3 The flow chart of the transmission scheduling algorithm delete the link $l_{p i}$ when all its traffic has been dealt with. Meanwhile, $F_{p}$ is renewed.

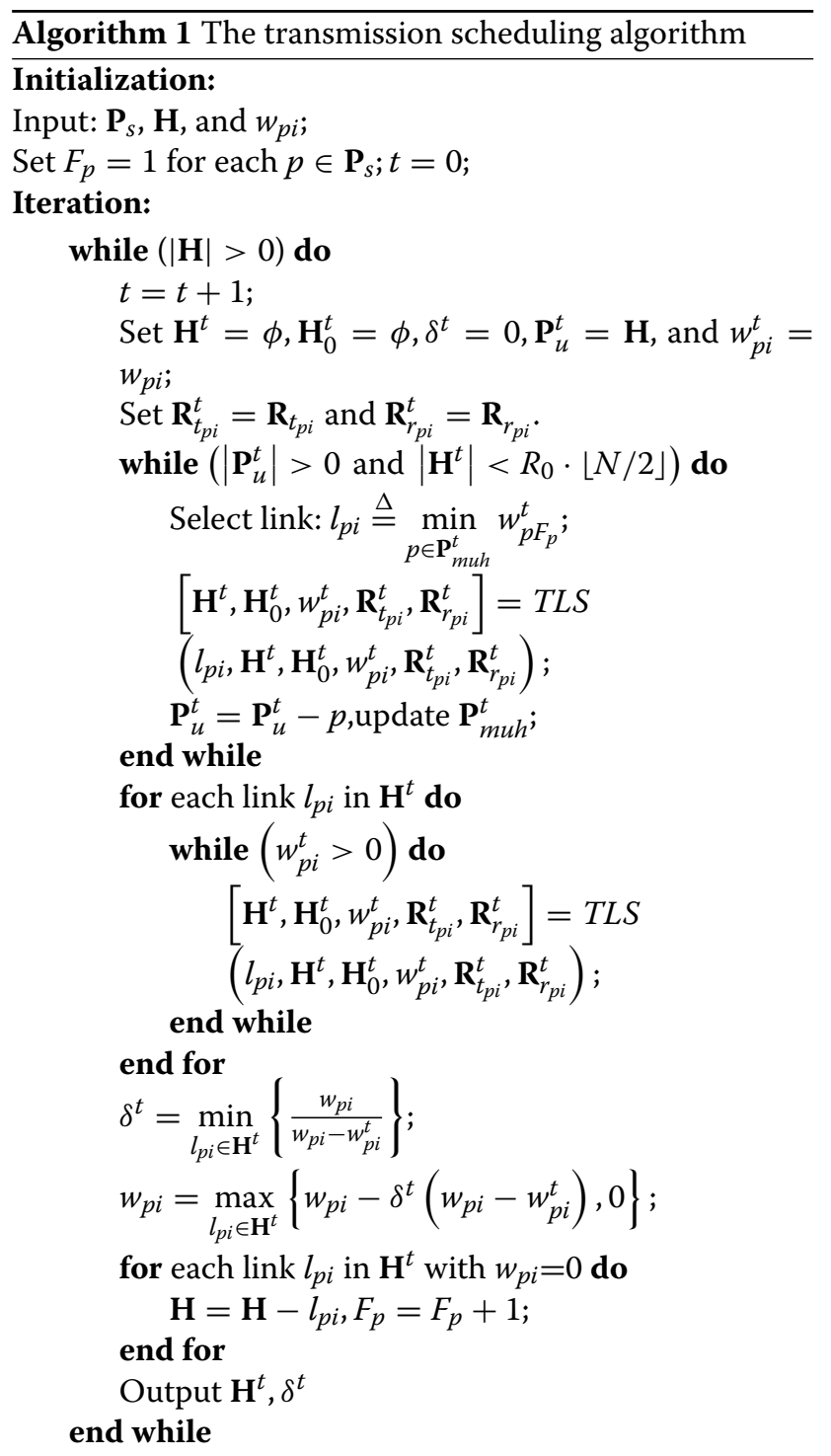

The pseudo code of the TLS algorithm is presented in Algorithm 2. It is used for selecting the tuple link $l_{p i}^{x}$ that brings minimum interference to the tuple links in $\mathbf{H}_{0}^{t}$. Line 1 examines the initial conditions for each selection. The algorithm starts when the transmitter and receiver own idle radios. Line 2 selects the tuple link $l_{p i}^{x}$ with minimum interference to the tuple links in $\mathbf{H}_{0}^{t}$ according to the cumulative interference level $I_{q j}^{y}$. With different $l_{p i}^{x} \notin \mathbf{H}_{0}^{t}$, it calculates the cumulative interference level $I_{q j}^{y}$ for each tuple link $l_{q j}^{y}$ in $\mathbf{H}_{0}^{t} \cup\left\{l_{p i}^{x}\right\}$ and obtains the maximum value. Thus, it gets a maximum cumulative interference level set. The tuple link $l_{p i}^{x_{0}}$ corresponding to 
the smallest value in the maximum cumulative interference level set will be selected. Line 3 gets the maximum cumulative interference level of links in $\mathbf{H}_{0}^{t} \cup\left\{l_{p i}^{x_{0}}\right\}$. Line 4 is a SINR condition to guarantee concurrent transmissions. Lines 6-8 renew $\mathbf{H}^{t}, \mathbf{H}_{0}^{t}, w_{p i}^{t}, \mathbf{R}_{t p i}^{t}$, and $\mathbf{R}_{r_{p i}}^{t}$ once the SINR condition is met.

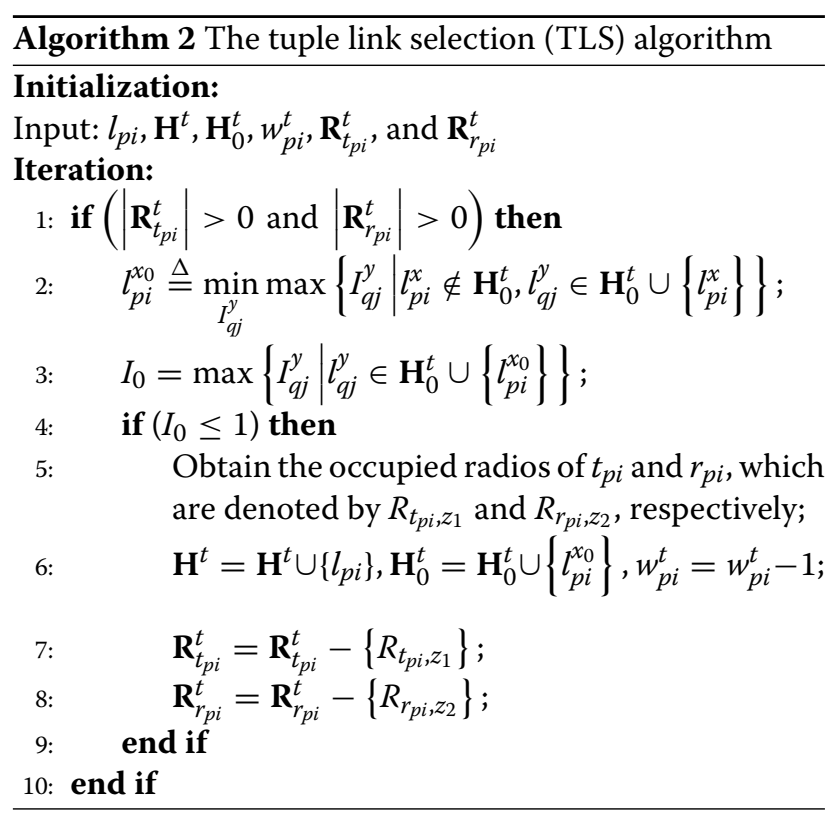

\subsection{Example}

As mentioned in 4.3, the interference level between tuple link $l_{p i}^{x}$ and $l_{q j}^{y}$, i.e., $W_{p i, q,}^{x, y}$, of case 6 is assumed to be 0.1 in Fig. 1. Applying this algorithm to the example in Fig. 1, it needs nine and five time slots in SRSC and MRMC (2-radio 2-channel) situation, respectively. The scheduling process in 2-radio 2-channel situation is illustrated in Fig. 4. The algorithm process is as follows:
Step 1: Initially, paths 2 and 3 both have three hops unscheduled, we have $F_{p}=1, p=1,2,3$, and $\mathbf{P}_{m u h}^{1}=$ $\{p \mid p=2,3\}$. According to the number of packets and rate of each link, we have $w_{2,1}^{1}=2$ and $w_{3,1}^{1}=1$. As $\delta^{1}=0$, link $l_{31}$ will be selected based on the criterion of line 4 in Algorithm 1 . Since $\mathbf{H}_{0}^{1}=\phi$, each tuple link of link $l_{31}$ is suitable to be added to $\mathbf{H}_{0}^{1}$ according to Algorithm 2 . We choose the one with minimum subscript of radios and channel, which is denoted as $\left(R_{A, 1}, R_{D, 1}, C_{1}\right)$. At the same time, link $l_{31}$ will be added to $\mathbf{H}^{1}$. Besides, $w_{3,1}^{1}$ will be updated and is given by $w_{3,1}^{1}=0$. Then we have $\mathbf{P}_{\text {muh }}^{1}=$ $\{p \mid p=2\}$. Similarly, link $l_{21}$ and tuple link $\left(R_{A, 2}, R_{C, 1}, C_{2}\right)$ will be added to $\mathbf{H}^{1}$ and $\mathbf{H}_{0}^{1}$, respectively. $w_{2,1}^{1}$ will be updated and is given by $w_{2,1}^{1}=1$. Meanwhile, $\mathbf{P}_{m u h}^{1}$ will also be updated and is given by $\mathbf{P}_{\text {muh }}^{1}=\{p \mid p=1\}$. However, according to algorithm 2 , link $l_{11}$ will not be added to $\mathbf{H}^{1}$ since the radios of transmitter $t_{11}$ (node $A$ ) are all occupied. The while loop between lines 3 and 7 will stop as $\left|\mathbf{P}_{u}^{t}\right|=0$.

Step 2: No tuple links will be added to $\mathbf{H}_{0}^{1}$ during the while loop between lines 8 and 11 since node $A$ has no free radios.

Step 3: We obtain the renewed $\delta^{1}, w_{2,1}$, and $w_{3,1}$, which are give by $\delta^{1}=1, w_{2,1}=1$, and $w_{3,1}=0$.

Step 4: $l_{3,1}$ will be removed from $\mathbf{H}$. $F_{3}$ will increase by 1 since all the traffic of $l_{3,1}$ has been cleared, which is given by $F_{3}=2$. The process of the second to fifth pairings are similar to the first pairing.

The scheduling results of our algorithm in SRSC situation are as follows: in the first pairing, link $A \rightarrow D$ transmits for one time slot; in the second pairing, links $A \rightarrow C$ and $D \rightarrow F$ transmit for two time slots; in the third pairing, links $D \rightarrow F, C \rightarrow E$, and $A \rightarrow B$ transmit for one time slot; in the fourth pairing, links $C \rightarrow E$ and $A \rightarrow B$ transmit for two time slots; in the fifth pairing, link $E \rightarrow B$ transmits for two time slots; in the sixth pairing, link $F \rightarrow B$ transmits for one time slot. However, it consumes 10 time slots with the algorithm in [1] in

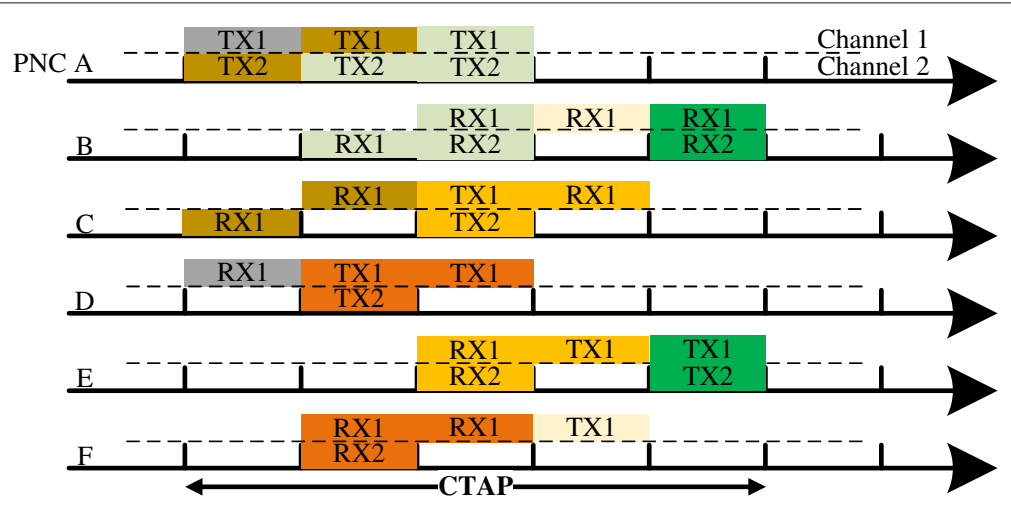

Fig. 4 An example of MPMH-MRMC operation applying the heuristic algorithm 
the SRSC situation. As analyzed in Section 4.3, the optimal solution takes nine and five time slots. Therefore, our heuristic scheme obtains results close to the optimal solution.

The path selection algorithm has the computational complexity of $O\left(N^{H_{\max }}\right)$, where $H_{\max }$ is the maximum number of hops for each selected path [1]. The computational complexity of the traffic distribution algorithm is negligible [1]. The transmission scheduling algorithm has the complexity of $O\left(N^{2}\right)$. Thus, the total complexity of the scheme is $O\left(N^{H_{\max }}+N^{2}\right)$, which is pseudo-polynomial. Thus, our scheme is practical for WPANs.

With the introduction of MPMH-MRMC, the control overhead will be increased since more scheduling information needs to be broadcasted to the nodes in the WPAN. Based on the directional MAC structure in Section 3.1, network synchronization and control messages for the $(m-1)$-th transmission requests are broadcasted in the $m$-th BP from the PNC. During the $m$-th CAP period, the $\mathrm{PNC}$ receives several transmission requests [3]. As mentioned in $[1,2]$, the $\mathrm{BP}$ or $\mathrm{CAP}$ can be completed by the PNC in one time slot. The PNC makes the path selection and scheduling decision for the $(m+1)$ th CTAP during the $m$-th CTAP. One CTAP contains nearly 1000 time slots. Generally, only a few time slots are needed for the PNC to complete path selection and schedule computation [1]. Considering that one frame consists of 1000 time slots $[1,3]$ and the Gbps transmission rates of the mmWave communications, the increase of the control overhead can be neglected.

\section{Simulation results}

In this section, we evaluate the performance of the proposed algorithm with respect to average delay and network throughput under two different traffic patterns. The algorithm applied in SRSC and MRMC situation are named MPMH-SRSC and MPMH-MRMC, respectively. We compare MPMH-SRSC and MPMH-MRMC with MPMH [1], MPMH2, and TDMA schemes. MPMH2 scheme applies the MPMH scheme [1] in MRMC situation by utilizing the TLS algorithm proposed in our paper. TDMA scheme supports only one active transmission in each time slot. In the MRMC situation, each node contains four radios, and there are four orthogonal channels in the network.

\subsection{Simulation setup}

In the simulation, a typical mmWave WPAN with 10 nodes is considered. It is assumed that all nodes are uniformly distributed in an area of $9 \times 9 \mathrm{~m}^{2}$. Considering link $l_{p i}$, the angle between the transmitting (receiving) direction and the center line of the beam of transmitter $t_{p i}$ (receiver $r_{p i}$ ) is assumed as $0^{\circ}$. Four transmission rates 2,4 , 6 , and $8 \mathrm{Gbps}$ are set based on the distance between WNs.
A typical physical layer parameter setting of mmWave WPANs is shown in Table 3. There are 10 flows in the network. The path selection and traffic distribution are obtained with the method in [1], and the maximum number of hops $H_{\max }$ for each selected path is set to 3 . We set the size of data packets to 1000 bytes. With the 2 Gbps transmission rate, a packet can be transmitted in a time slot [2]. The length of simulation is set to $5 \times 10^{4}$ time slots. A frame consists of 1000 time slots. The delay threshold is $2.5 \times 10^{4}$ time slots, and packets with delay larger than the threshold will be discarded. The traffic mode is assumed to be Poisson process.

Poisson process: packets of each flow are generated following a Poisson process with arrival rate $\lambda_{i}$. The traffic load ratio given by $T_{l}$ can be defined as

$$
T_{l}=\left(L \cdot \sum_{i=1}^{V} \lambda_{i}\right) / R,
$$

where $L$ is the size of data packets in bit, $V$ is the number of flows, and $R$ is set to be 2 Gbps.

In the simulation, two traffic patterns are adopted.

1) Uniform traffic: All flows have the same arrival rate $\lambda$, i.e., $\lambda_{i}=\lambda$, which is adopted in [1].

2) Random traffic: The arrival rate of flow $i, \lambda_{i}$, is randomly generated.

The system performance is evaluated by the following two metrics:

1) Average delay: The average delay of received packets of all flows, and the unit is time slot. The delay caused by $\mathrm{BP}$ and CAP is not calculated.

2) Network throughput: The number of successfully transmitted packets of all flows during the entire simulation, and the unit is packet. A packet will be counted as a successfully transmitted packet when its delay is no more than the threshold.

\subsection{Network throughput with different traffic load ratio}

We evaluate the network throughput of five protocols with different traffic load ratio in Fig. 5. It can be observed that MPMH-SRSC outperforms MPMH and TDMA in all cases, and the gap between MPMH-SRSC

Table 3 Simulation parameters

\begin{tabular}{lll}
\hline Symbol & Description & Value \\
\hline$W$ & Channel bandwidth & $1200 \mathrm{MHz}$ \\
$P_{t}$ & Transmission power & $0.1 \mathrm{~mW}$ \\
$N_{0}$ & Background noise & $-114 \mathrm{dBm} / \mathrm{MHz}$ \\
$\rho$ & Path loss exponent & 2 \\
$A_{\text {LOS }}$ & LOS path loss constant & $32.5 \mathrm{~dB}$ \\
$\theta_{-3 \mathrm{~dB}}$ & The antennas HPBW & $60^{\circ}$ \\
$T_{\text {slot }}$ & Slot duration & $5 \mu \mathrm{s}$ \\
\hline
\end{tabular}



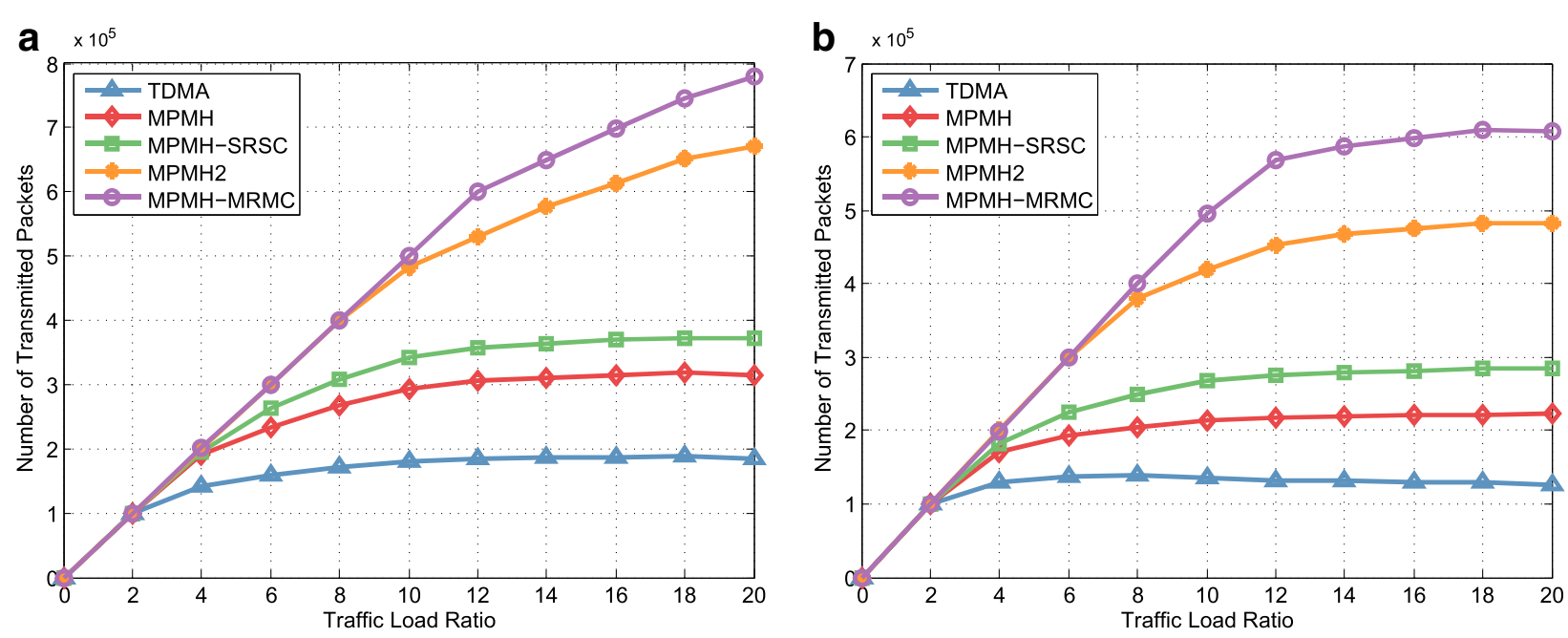

Fig. 5 Network throughput of the five MAC protocols under different traffic load ratios. a Uniform traffic. b Random traffic

and MPMH is more significant under heavy load. Under light load, the delay is small, and all the arrived packets can be transmitted successfully when applying MPMH-SRSC and MPMH. Thus, the throughput of MPMH-SRSC and MPMH increase linearly under light load. Compared with MPMH, MPMH-SRSC increases the network throughput with the traffic load ratio from 10 to 20 on average by about $17.09 \%$ under uniform traffic and $27.09 \%$ under random traffic, respectively.

The increment of the random traffic is larger than that of the uniform traffic. The reasons are as follows. Under uniform traffic, the transmission durations of links in a pairing are diverse in MPMH [1]. The diversities bring about the waste of time slots in a pairing. The differences among the transmission durations of links in a pairing under random traffic are worse, which result in further degradations in the throughput performance. However, the transmission durations of links in a pairing are the same under both traffic patterns in MPMH-SRSC. Thus, it outperforms the MPMH under uniform traffic and has greater performance increment under random traffic.

With the introduction of MRMC, MPMH-MRMC can better utilize MPMH than the MPMH-SRSC, and the throughput of MPMH-MRMC increases linearly even under heavy traffic load. With the traffic load ratio from 10 to 20, MPMH-MRMC surpasses MPMH-SRSC by about $82.34 \%$ under uniform traffic and $108.29 \%$ under random traffic, respectively. Compared with MPMH2, MPMH-MRMC increases the network throughput by about $14.09 \%$ under uniform traffic and $25.8 \%$ under random traffic on average with the traffic load ratio from 12 to 20. The reason is the same with the one in SRSC situation. When the traffic load ratio is over 3 , the number of discarded packets in TDMA exceeds MPMH and MPMHSRSC, which results in smaller network throughput in TDMA. Since the delay threshold is larger than the frame length, the packets that cannot be scheduled in current frame will be transmitted in the next frame. With the increase of the traffic load ratio, the number of packets successful transmitted in the system becomes stable and the network throughput curves become flat.

\subsection{Network throughput with different number of flows with multi-path}

Under random traffic, the network throughput achieved by the four protocols with different number of flows with multi-path is plotted in Fig. 6. The flows transmitted through MPMH are selected according to traffic demand intensity in [1]. With the same mean traffic load ratio of a flow, the throughput of MPMH-MRMC increases at most about $1.39 \%$ when traffic load ratio is 25 with 10 flows and $44.76 \%$ when the traffic load ratio is 10 with 4 flows.

Considering that the paths of all flows construct a directed graph, the degree of one node will increase when introducing the MPMH. With dense flows, MPMHMRMC has sufficiently utilized MRMC in the SPSH situation. When the flows are transmitted through multi-path, the degree of a node is beyond the ability of the MRMC, which results in the degradation of the network throughput. However, with sparse flows in the network, the MRMC of a node is underutilized until more flows are transmitted through MPMH. MPMH-SRSC and MPMH are employed in the SRSC situation, one node has fully exploited its radio even when the SPSH is applied. As a result, the performance of MPMH-SRSC and MPMH decrease rapidly with the increase of number of flows 

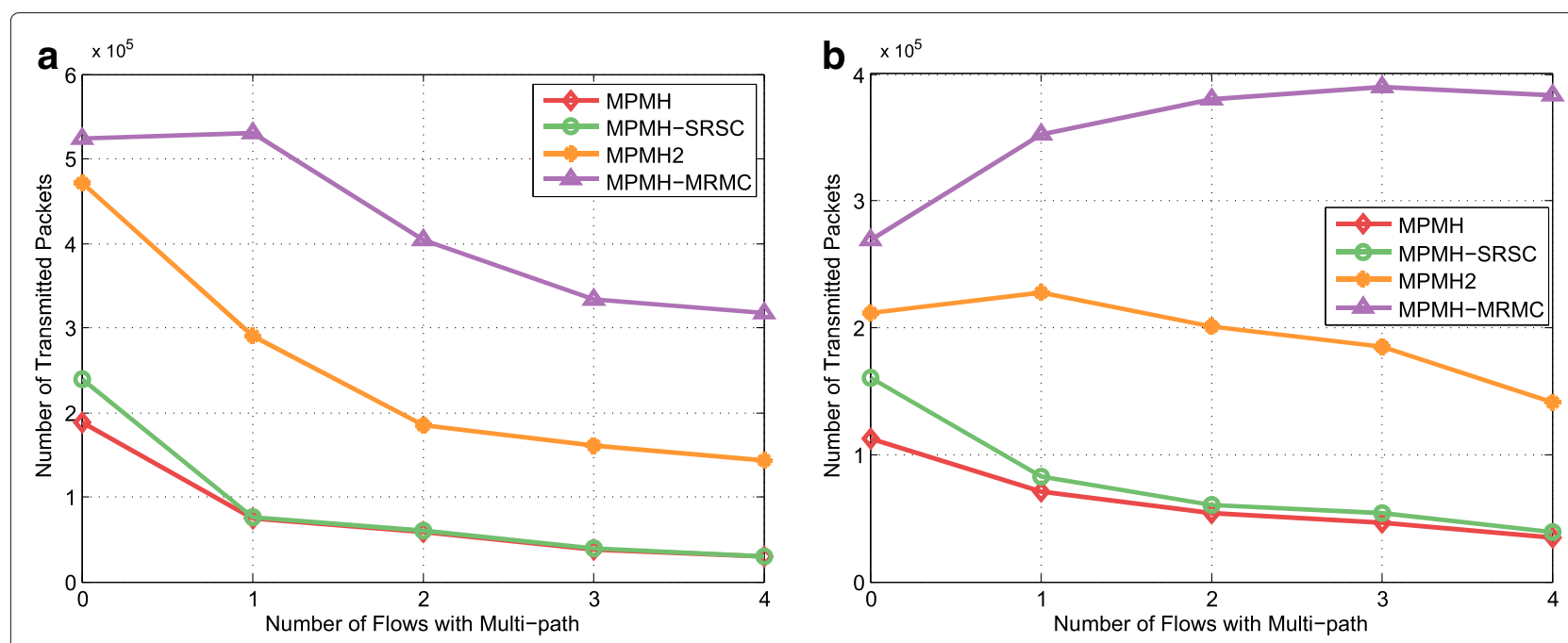

Fig. 6 Network throughput of the four MAC protocols under a different number of flows with multi-path. a Traffic load ratio is 25 with 10 flows. b Traffic load ratio is 10 with 4 flows

transmitted through MPMH in both situations. For the similar reason, the performance of MPMH2 decreases when traffic load ratio is 25 with 10 flows and increases about $7.58 \%$ when traffic load ratio is 10 with 4 flows.

\subsection{Average delay with different traffic load ratio}

The average delay of five protocols with different traffic load ratio are presented in Fig. 7. We can observe that the delay of the five protocols increases with the increase of traffic load ratio. The arrived packets can be transmitted in a short time under light load. Thus, the delay is small in this case. With the traffic load ratio from 3 to 20, compared with MPMH, MPMH-SRSC decreases the average transmission delay by about $16.53 \%$ under uniform traffic and $32.06 \%$ under random traffic on average. The increment of random traffic is larger than uniform traffic. The reason is identical with the one in network throughput.

As mentioned in Section 6.2, MPMH-MRMC can better utilize MPMH than the MPMH-SRSC, which results in the decrease of delay. Compared with MPMH-SRSC, the average transmission delay of MPMH-MRMC decreases by about $74.20 \%$ under uniform traffic and $70.91 \%$ under random traffic with the traffic load ratio from 10 to 20. With the traffic load ratio from 10 to 20, MPMHMRMC outperforms MPMH2 by about $29.21 \%$ under
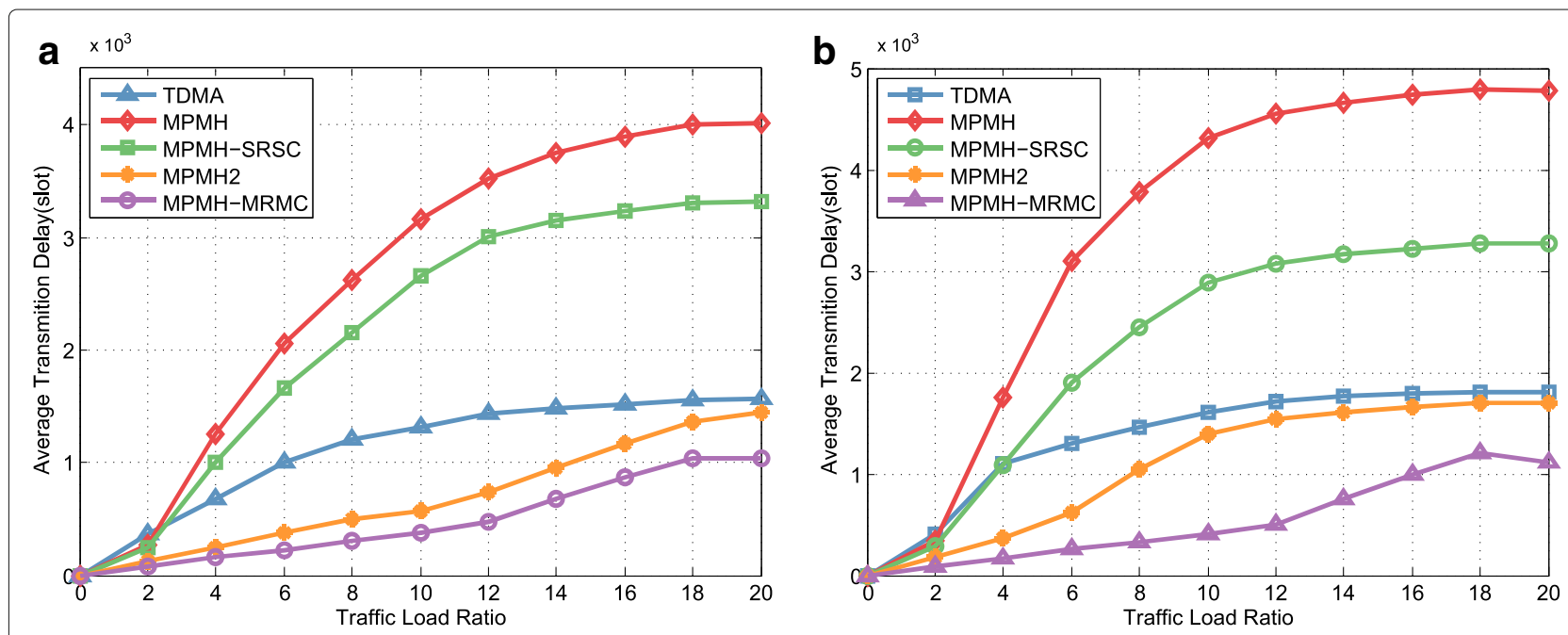

Fig. 7 Average transmission delay of the five MAC protocols under different traffic load ratios. a Uniform traffic. b Random traffic 

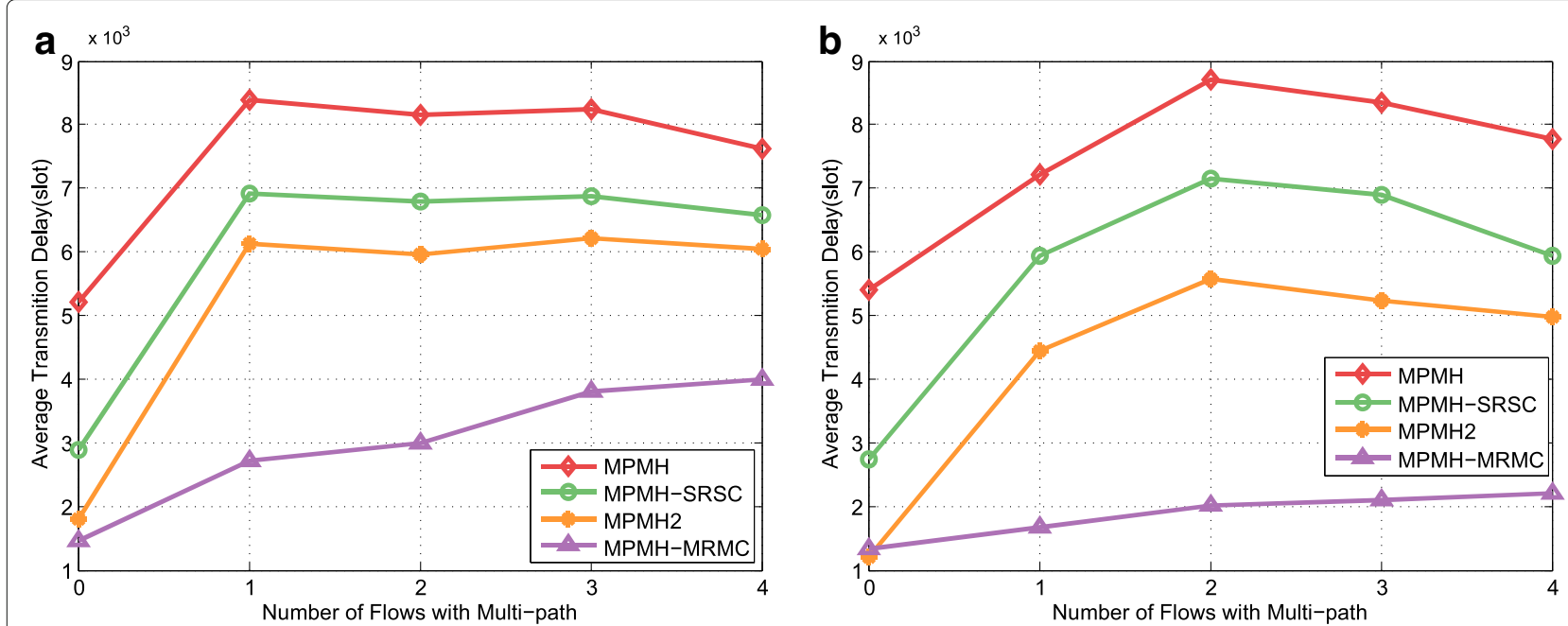

Fig. 8 Average transmission delay of the four MAC protocols under a different number of flows with multi-path. a Traffic load ratio is 25 with 10 flows. b Traffic load ratio is 10 with 4 flows

uniform traffic and $49.04 \%$ under random traffic on average, respectively. The reason is identical with the one in network throughput. When the traffic load ratio is over 3 , the number of discarded packets in TDMA exceeds MPMH and MPMH-SRSC, which leads to lower average delay in TDMA.

\subsection{Average delay with different number of flows with multi-path}

In Fig. 8, we plot the average delay achieved by the four protocols with different number of flows with multi-path under random traffic. We can observe that the average delay increases when the flow with multi-path is introduced, and it decreases a little when more flows are applied with multi-path. As mentioned in Section 6.3, one node has fully exploited its radio even when the SRSC is applied in MPMH-SRSC and MPMH. Identical with the reason in network throughput, the performance of average delay degrades when multi-path is introduced. However, the throughput decreases continuously with the increase of numbers of the flows with multi-path, which results in the little decrease of the average delay. The reason above can also be used to explain the performance of average delay of MPMH2.

Compared with MPMH, MPMH-SRSC decreases the delay by about $21.86 \%$ when the traffic load ratio is 25 with 10 flows and $25.01 \%$ when traffic load ratio is 10 with 4 flows, respectively. MPMH-MRMC surpasses MPMHSRSC by about 49.96 and $66.72 \%$ correspondingly. Besides, MPMH-MRMC outperforms MPMH2 by about 39.33 and $49.04 \%$. The results are consistent with the ones of the network throughput.

\section{Conclusions}

In this paper, we have proposed a scheduling algorithm for mmWave WPANs, which boosts the potential of spatial reuse by concurrent transmissions scheduling through MPMH-MRMC. It can sufficiently improve the performance of network throughput and average delay. The proposed heuristic algorithm can achieve the results close to the optimal solution with the complexity of $O\left(N^{H_{\max }}+N^{2}\right)$. Compared with MPMH, MPMH-SRSC enhances the performance of network throughput and delay by about 22.09 and $24.30 \%$ on average, respectively. MPMH-MRMC outperforms MPMH-SRSC in terms of the network throughput and delay by about 95.32 and $72.56 \%$ on average. Performance under different number of flows transmitted through MPMH indicates the number of flows transmitted through MPMH should be selected according to the flow density to optimize network performance.

\section{Funding}

This work was sponsored by the National Natural Science Foundation of China (61631003).

\section{Availability of data and materials Not applicable.}

\section{Authors' contributions}

All authors contribute to the concept, the design and developments of the theory analysis and heuristic algorithm, and the simulation results in this manuscript. All authors read and approved the final manuscript.

\section{Competing interests}

The authors declare that they have no competing interests.

\section{Publisher's Note}

Springer Nature remains neutral with regard to jurisdictional claims in published maps and institutional affiliations. 


\section{Author details}

${ }^{1}$ Key Lab. of Universal Wireless Communications Ministry of Education, Beijing University of Posts and Telecommunications (BUPT), Haidian Dist. Xitucheng Rd., Beijing, People's Republic of China. ${ }^{2}$ China Academy of Information and Communications Technology, No.52, Huayuanbei Rd., Haidian Dist., Beijing, People's Republic of China.

Received: 2 May 2017 Accepted: 7 March 2018

Published online: 20 March 2018

\section{References}

1. Y Niu, C Gao, Y Li, D Jin, L Su, D Wu, Boosting spatial reuse via multiple-path multihop scheduling for directional mmWave WPANs. IEEE Trans. Veh. Technol. 65(8), 6614-6627 (2016)

2. IK Son, S Mao, MX Gong, Y Li, in Proc. IEEE International Conference on Computer Communications (INFOCOM). On frame-based scheduling for directional mmWave WPANs, (2012), pp. 2149-2157

3. J Qiao, LX Cai, X Shen, JW Mark, in Proc. IEEE International Conference on Communications (ICC). STDMA-based scheduling algorithm for concurrent transmissions in directional millimeter Wave Networks, (2012), pp. 5221-5225

4. Z Yan, B Li, X Zuo, M Yang, in Proc. IEEE Wireless Communications and Networking Conference (WCNC). A Heuristic clique based STDMA scheduling algorithm for spatial concurrent transmission in mmWave networks, (2015), pp. 1036-1041

5. J Qiao, LX Cai, X Shen, JW Mark, Enabling multi-hop concurrent transmissions in $60 \mathrm{GHz}$ wireless personal area networks. IEEE Trans. Wireless Commun. 10(11), 3824-3833 (2011)

6. MX Cheng, Q Ye, L Cai, Rate-adaptive concurrent transmission scheduling schemes for WPANs with directional antennas. IEEE Trans. Veh. Technol. 64(9), 4113-4123 (2015)

7. P Xu, H Chu, in Proc. IEEE Wireless Communications and Networking Conference (WCNC). A novel link scheduling strategy for concurrent transmission in mmWave WPANs based on beamforming information, (2014), pp. 1709-1714

8. L Zhou, X Cao, L Liu, L Cai, in Proc. IEEE International Conference on Communications (ICC). On capacity optimization in multi-radio multi-channel wireless networks with directional antennas, (2015), pp. 3745-3750

9. R Mudumbai, S Singh, U Madhow, in Proc. IEEE International Conference on Computer Communications (INFOCOM). Medium access control for $60 \mathrm{GHz}$ outdoor mesh networks with highly directional links, (2009), pp. $2871-2875$

10. C-S Sum, Z Lan, R Funada, J Wang, T Baykas, M Rahman, H Harada, Virtual time-slot allocation scheme for throughput enhancement in a millimeter-wave multi-Gbps WPAN system. IEEE J. Sel. Areas Commun. 27(8), 1379-1389 (2009)

11. CS Sum, Z Lan, MA Rahman, J Wang, T Baykas, R Funada, H Harada, S Kato, in Proc. IEEE Global Telecommunications Conference (GLOBECOM). A multi-Gbps millimeter-wave WPAN system based on STDMA with heuristic scheduling, (2009), pp. 1-6

12. LX Cai, L Cai, X Shen, JW Mark, REX: a randomized exclusive region based scheduling scheme for mmWave WPANs withdirectional antenna. IEEE Trans. Wirel. Commun. 9(1), 113-121 (2010)

13. X An, R Hekmat, in IEEE Vehicular Technology Conference (VTC). Directional MAC protocol for millimeter wave based wireless personal area networks, (2008), pp. 1636-1640

14. CW Pyo, F Kojima, J Wang, H Harada, S Kato, MAC Enhancement for high speed communications in the 802.15.3c mmWave WPAN. Wireless Pers. Commun. 51(4), 825-841 (2009)

15. P Dutta, V Mhatre, D Panigrahi, R Rastogi, in Proc. IEEE International Conference on Computer Communications (INFOCOM). Joint routing and scheduling in multi-hop wireless networks with directional antennas, (2010), pp. 1-5

16. M Kim, Y Kim, W Lee, Resource allocation scheme for millimeter wave-based WPANs using directional antennas. ETRI J. 36(3), 385-395 (2014)

17. M Kim, SE Hong, Y Kim, J Kim, Analysis of resource assignment for directional multihop communications in mm-Wave WPANs. ETRI J. 35(1), $120-130$ (2013)
18. H Shokri-Ghadikolaei, L Gkatzikis, C Fischione, in Proc. IEEE International Conference on Communications (ICC). Beam-searching and transmission scheduling in millimeter wave communications, (2015), pp. 9-14

19. H Su, X Zhang, in Proc. Global Telecommunications Conference (GLOBECOM). Joint link scheduling and routing for directional-antenna based $60 \mathrm{GHz}$ wireless mesh networks, (2009), pp. 1-6

20. M Bilal, M Kang, SC Shah, S-G Kang, Time-slotted scheduling schemes for multi-hop concurrent Transmission in WPANs with directional antenna. ETRI J. 36(3), 374-384 (2014)

21. S Kompella, S Mao, YT Hou, HD Sherali, On path selection and rate allocation for video in wireless Mesh Networks. IEEE/ACM Trans. Netw. 17(1), 212-224 (2009)

\section{Submit your manuscript to a SpringerOpen ${ }^{\circ}$ journal and benefit from:}

- Convenient online submission

- Rigorous peer review

- Open access: articles freely available online

- High visibility within the field

- Retaining the copyright to your article

Submit your next manuscript at $>$ springeropen.com 